\title{
Analysis and Experimental Validation of an HTS Magnetic Suspension and Linear Synchronous Propulsion Prototype
}

\author{
Jianxun Jin ${ }^{\mathrm{a}, *}$, Luhai Zheng ${ }^{\mathrm{a}}$, Youguang Guo ${ }^{\mathrm{b}}$, Wei Xu ${ }^{\text {b,* }}$, and Jianguo Zhu ${ }^{\mathrm{b}}$ \\ ${ }^{1}$ Center of Applied Superconductivity and Electrical Engineering, University of Electronic Science and Technology of China, Chengdu 611731, China \\ ${ }^{2}$ Faculty of Engineering and Information Technology, University of Technology Sydney, Sydney, PO Box 123, NSW 2007, Australia
}

\begin{abstract}
The electromagnetic design, performance analysis and experimental measurements of a high temperature superconducting (HTS) magnetic suspension and propulsion system using an HTS linear synchronous motor (HTSLSM) drive have been carried out. Different magnetization methods have been employed to study the magnetic flux trapping characteristics of the HTS bulk magnets to validate the HTS technology for the HTSLSM application. Three different types of permanent magnet guideways (PMGs) for the HTS magnetic suspension have been developed, and their field distribution characteristics have also been analyzed by using an equivalent current sheet (ECS) method and verified by practical measurements. The levitation forces based on the three PMGs have been calculated and compared. An optimal PMG has been obtained as to provide the largest levitation force and better ratio (levitation force) / (permanent magnet volume). Based on comprehensive numerical analysis and experimental testing of a prototype, typical performances of the HTSLSM have been obtained, including the thrust characteristics versus the frequency, phase current and air-gap length, which would benefit the electromagnetic design and the control scheme development for the HTSLSM.
\end{abstract}

PACS: 85.25.Ly

Keywords: High temperature superconductors, HTS linear synchronous motor, HTS magnetic levitation, Pulse magnetization, Field-cooled magnetization, Trapped field attenuation

\section{Introduction}

Various kinds of high temperature superconducting (HTS) suspension and linear propulsion systems using HTS tapes or HTS bulk magnets have been developed. These suspension systems can be categorized as the electro-dynamic (repulsion) suspension (EDS) using HTS coil magnets [1-4], the electro-magnetic (attraction) suspension (EMS) using HTS coils [5, 6], and the HTS magnetic (repulsion) suspension using field-cooled HTS bulks [7-9]. Among them, the HTS magnetic suspension system is the simplest in structure using HTS bulks on the moving low temperature vessels and permanent magnet guideways (PMGs) as the track, and has the capabilities of self-levitation and self-guidance without using any feedback control.

The HTS linear motors developed so far can be mainly classified according to their principle and structure as the HTS linear reluctance motor (LRM) with ordinary copper windings on the primary (stator) and zero-field-cooled (ZFC) HTS bulk poles on the secondary (mover) $[10,11]$, the HTS linear synchronous motor (HTSLSM) with ordinary copper windings on the primary and HTS bulk or coil magnets on the secondary [1-4,12-15-16], and the HTSLSM or linear induction motor (LIM) with HTS windings on the primary $[16-17,18]$. These HTS linear motors can be further divided into single-sided, double-sided, and cylindrical types according to their structures. Compared to the conventional linear motors, the HTS linear motors have a number of potential advantages, such as small size, high thrust, low weight, and high efficiency because of using zero-resistance HTS tapes or strong-pinning HTS bulks with high trapped magnetic fields.

A compound model of an HTS magnetic suspension and HTS linear synchronous propulsion system has been proposed and developed. The system is driven by a long-primary single-sided HTSLSM with HTS bulk magnet secondary, and the secondary mover is levitated by the HTS magnetic suspension systems on both sides of the HTSLSM. The propulsion system can run with stable thrust without any sliding friction, and have the self-levitation and self-guidance functions in both static and dynamic operating states. As the results of using HTS both for propulsion and suspension, the thrust of the compound HTSLSM system is higher than the systems using conventional linear motors or HTS LRM for propulsion because of HTS bulk magnets having higher trapped magnetic fields.

To validate the performance characteristics of the prototype, a thrust measurement system has been developed to measure the lock-mover thrust of the HTSLSM, where the amplitude of which is the maximum thrust $F_{\text {em_max }}$. In this work, the $F_{\text {em_max }}$ characteristics have been obtained by measuring the amplitude of the lock-mover thrust under various conditions.

The levitation force, $F_{\mathrm{Lev}}$, and the guidance force, $F_{\mathrm{Gui}}$, are two principal performance indicators for the HTS magnetic suspension system. While $F_{\text {Gui }}$ depends on the trapped flux in the HTS bulk, $F_{\text {Lev }}$ is linearly proportional to the gradient of the magnetic field of PMG. Therefore it is important to design a PMG with high magnetic field gradient. Three types of PMGs have been designed for the HTS magnetic suspension system, and their magnetic field distribution characteristics have been analyzed and compared both by equivalent current sheet (ECS) models and measurements so as to obtain the optimum PMG for the HTS magnetic suspension system. It indicates that the ECS method could provide a general method for the calculation of the field distribution of a PMG.

\section{The System}

Fig.1 illustrates schematically the developed HTS magnetic suspension and propulsion system with an HTSLSM drive, where the HTSLSM is located in the middle between two HTS magnetic suspension sub-systems. Three types of PMGs are designed, as shown in Fig. 2, with (a) Surface mounted (\#1), (b) Flux concentration (\#2), and (c) Halbach array PMGs (\#3), respectively, where the arrows indicate the magnetization directions of the $\mathrm{NdFeB}$ permanent magnets (PMs). The values of $w_{1}, w_{2}, w_{3}$ and $h_{\mathrm{pm}}$ occurring in these figures are listed in Table 1.

The primary (stator) and the secondary (mover) of the HTSLSM are made of three-phase copper windings and magnetized YBCO(123) HTS bulk magnets, respectively, and the magnets are fixed in cryogenic vessels with alternating $\mathrm{N}$ and $\mathrm{S}$ poles along the longitudinal direction, and same poles along the transverse direction to reach the suitable width. Main parameters of the HTSLSM are listed in Table 1 -2. 


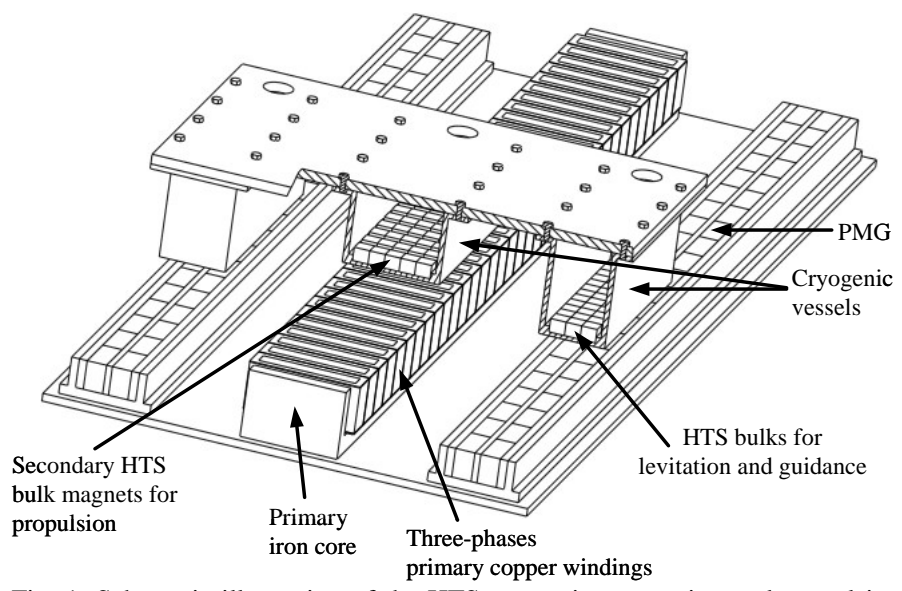

Fig. 1. Schematic illustration of the HTS magnetic suspension and propulsion system with an HTSLSM drive.

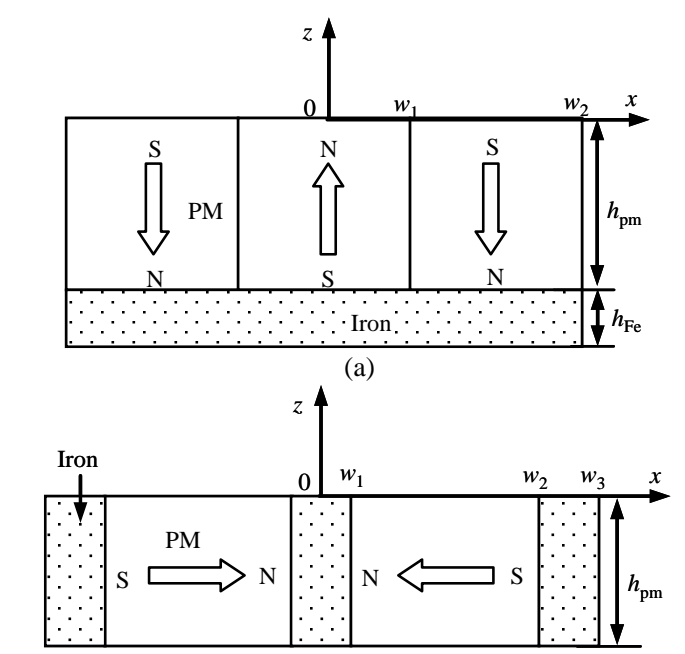

(b)

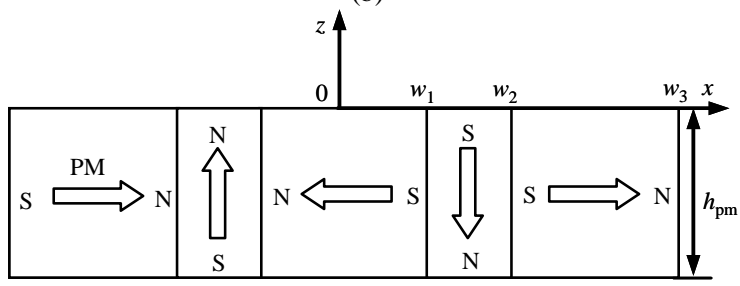

(c)

Fig. 2. Schematic structures of PMGs. (a) Surface mounted PMG (\#1), (b) Flux concentration PMG (\#2), and (c) Halbach array PMG (\#3).

Table 1

Main dimensions of transversal section of the PMGs

\begin{tabular}{llll}
\hline \hline & & $w_{1}$ & $10 \mathrm{~mm}$ \\
\#1 PMG & PMs & $w_{2}$ & $30 \mathrm{~mm}$ \\
& & width $\left(w_{2}-w_{1}\right)$ & $20 \mathrm{~mm}$ \\
& & height $\left(h_{\mathrm{pm}}\right)$ & $25 \mathrm{~mm}$ \\
& \multirow{3}{*}{ Soft iron } & width $\left(2 w_{2}\right)$ & $60 \mathrm{~mm}$ \\
& & height $\left(h_{\mathrm{Fe}}\right)$ & $8 \mathrm{~mm}$ \\
& & $w_{1}$ & $4 \mathrm{~mm}$ \\
& & $w_{2}$ & $29 \mathrm{~mm}$ \\
\#2 PMG & PMs & $w_{3}$ & $37 \mathrm{~mm}$ \\
& & width $\left(w_{2}-w_{1}\right)$ & $25 \mathrm{~mm}$ \\
& & height $\left(h_{\mathrm{pm}}\right)$ & $20 \mathrm{~mm}$ \\
& Soft iron & width $\left(2 w_{1}\right)$ & $8 \mathrm{~mm}$ \\
& $w_{1}$ & height $\left(h_{\mathrm{pm}}\right)$ & $20 \mathrm{~mm}$ \\
\#3 PMG & $w_{2}$ & & $10 \mathrm{~mm}$ \\
& $w_{3}$ & & $20 \mathrm{~mm}$ \\
& $h_{\mathrm{pm}}$ & & $20 \mathrm{~mm}$ \\
\hline \hline
\end{tabular}

Table 12

Main parameters of the HTSLSM

\begin{tabular}{lll}
\hline \hline Part & Item & Value \\
& Width & $150 \mathrm{~mm}$ \\
& Height & $140 \mathrm{~mm}$ \\
Primary & Length & $2220 \mathrm{~mm}$ \\
& tooth length $\left(l_{\mathrm{t}}\right)$ & $10 \mathrm{~mm}$ \\
& tooth width $\left(w_{\mathrm{t}}\right)$ & $150 \mathrm{~mm}$ \\
\hline \hline
\end{tabular}

\begin{tabular}{lll}
\hline \hline & Pole pitch $(\tau)$ & $45 \mathrm{~mm}$ \\
& Number of slots $(Q)$ & 72 \\
\multirow{5}{*}{ Air gap } & Pair poles $(p)$ & 24 \\
& Length $(g)$ & $5 \mathrm{~mm}$ \\
& HTS bulk magnet length $\left(l_{\mathrm{s}}\right)$ & $30 \mathrm{~mm}$ \\
& HTS bulk magnet width $\left(w_{\mathrm{s}}\right)$ & $30 \mathrm{~mm}$ \\
& HTS bulk magnet height $\left(h_{s}\right)$ & $15 \mathrm{~mm}$ \\
\multirow{5}{*}{ Secondary } & Trapped magnetic flux intensity $\left(B_{\text {trap }}\right)$ & $0.5 \mathrm{~T}$ \\
& Relative permeability $\left(\mu_{r}\right)$ & 0.4 \\
& Number of HTS bulk magnets along & 6 \\
& longitudinal direction & \\
& Number of HTS bulk magnets along & 5 \\
Operational & transverse direction & $1 \mathrm{kN}$ \\
parameters & Maximal thrust $\left(F_{\text {em_max }}\right)$ & $60 \mathrm{~N} / \mathrm{A}$ \\
\hline \hline
\end{tabular}

\section{Levitation and Guidance Forces}

\subsection{PMG Models}

The ECS method, which models a PM by a pair of current sheets of infinitesimal thickness, is used to derive the three different PMG models by ignoring the back iron. Fig.3 illustrates the sheet current directions corresponding to the three PMGs shown in Fig.2. The magnetic flux density at a given point $(x, z)$ can be obtained by summing up the contributions of each current sheet, which is calculated by the Biot-Savart law.

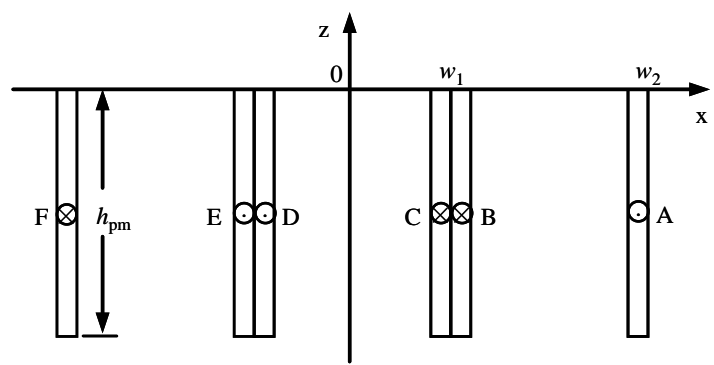

(a)

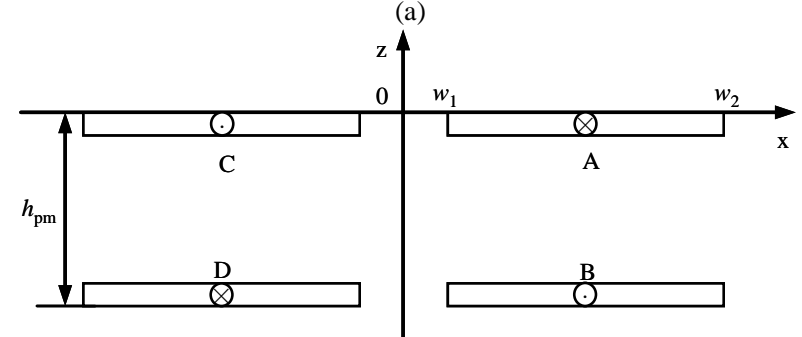

(b)

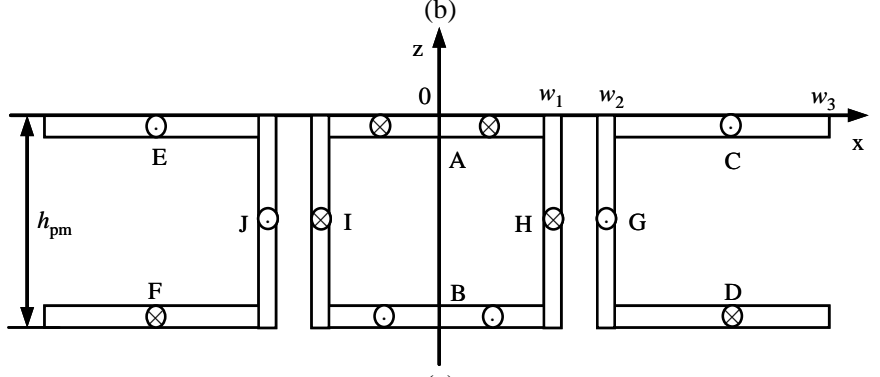

(c)

Fig. 3. ECS models of PMGs: (a) Surface mounted PMG (\#1), (b) Flux concentration PMG (\#2), and (c) Halbach array PMG (\#3).

For the Halbach array PMG with current sheets as shown in Fig.3(c), the flux density due to current sheet A can be obtained by integrating the contributions of elemental currents $d I=-M_{0} d z$, where $M_{0}$ is the magnetization intensity of PM, and $d z$ an infinitesimal length along the $\mathrm{z} z$ direction, as the following

$$
\begin{gathered}
B_{x_{-} \mathrm{A}}=\frac{\mu_{0}\left(-M_{0}\right)}{4 \pi}\left(2 \arctan \frac{w_{1}-x}{z}-2 \arctan \frac{-w_{1}-x}{z}\right) \\
B_{z_{-} \mathrm{A}}=\frac{\mu_{0}\left(-M_{0}\right)}{4 \pi} \ln \frac{\left(x+w_{1}\right)^{2}+z^{2}}{\left(x-w_{1}\right)^{2}+z^{2}}
\end{gathered}
$$

where $\mu_{0}$ is the permeability of vacuum.

Similarly, the magnetic flux density at point $(x, z)$ generated by other current sheets can also be calculated, and the total flux density 
generated by all current sheets at a given point $(x, z)$ can be derived as

$$
\begin{aligned}
B_{x}= & \frac{\mu_{0} M_{0}}{4 \pi}\left[-2 \arctan \frac{w_{1}-x}{z}+2 \arctan \frac{-w_{1}-x}{z}+2 \arctan \frac{w_{1}-x}{h_{\mathrm{pm}}+z}-2 \arctan \frac{-w_{1}-x}{h_{\mathrm{pm}}+z}+2 \arctan \frac{w_{3}-x}{z}-2 \arctan \frac{w_{2}-x}{z}\right. \\
& -2 \arctan \frac{w_{3}-x}{z+h_{\mathrm{pm}}}+2 \arctan \frac{w_{2}-x}{z+h_{\mathrm{pm}}}-2 \arctan \frac{-w_{3}-x}{z}+2 \arctan \frac{-w_{2}-x}{z}+2 \arctan \frac{-w_{3}-x}{z+h_{\mathrm{pm}}}-2 \arctan \frac{-w_{2}-x}{z+h_{\mathrm{pm}}} \\
+ & \left.\ln \frac{\left(w_{2}-x\right)^{2}+\left(z+h_{\mathrm{pm}}\right)^{2}}{\left(w_{2}-x\right)^{2}+z^{2}}-\ln \frac{\left(w_{1}-x\right)^{2}+\left(z+h_{\mathrm{pm}}\right)^{2}}{\left(w_{1}-x\right)^{2}+z^{2}}-\ln \frac{\left(-w_{1}-x\right)^{2}+\left(z+h_{\mathrm{pm}}\right)^{2}}{\left(-w_{1}-x\right)^{2}+z^{2}}+\ln \frac{\left(-w_{2}-x\right)^{2}+\left(z+h_{\mathrm{pm}}\right)^{2}}{\left(-w_{2}-x\right)^{2}+z^{2}}\right] \\
B_{z}= & \frac{\mu_{0} M_{0}}{4 \pi}\left[-\ln \frac{\left(x+w_{1}\right)^{2}+z^{2}}{\left(x-w_{1}\right)^{2}+z^{2}}+\ln \frac{\left(x+w_{1}\right)^{2}+\left(z+h_{\mathrm{pm})^{2}}\right.}{\left(x-w_{1}\right)^{2}+\left(z+h_{\mathrm{pm}}\right)^{2}}+\ln \frac{\left(x-w_{2}\right)^{2}+z^{2}}{\left(x-w_{3}\right)^{2}+z^{2}}-\ln \frac{\left(x-w_{2}\right)^{2}+\left(z+h_{\mathrm{pm}}\right)^{2}}{\left(x-w_{3}\right)^{2}+\left(z+h_{\mathrm{pm}}\right)^{2}}\right. \\
& +\ln \frac{\left(x+w_{3}\right)^{2}+z^{2}}{\left(x+w_{2}\right)^{2}+z^{2}}-\ln \frac{\left(x+w_{3}\right)^{2}+\left(z+h_{\mathrm{pm}}\right)^{2}}{\left(x+w_{2}\right)^{2}+\left(z+h_{\mathrm{pm}}\right)^{2}}-2 \arctan \frac{-z}{w_{2}-x}+2 \arctan \frac{-h_{\mathrm{pm}}-z}{w_{2}-x}+2 \arctan \frac{-z}{w_{1}-x} \\
& \left.-2 \arctan \frac{-h_{\mathrm{pm}}-z}{w_{1}-x}+2 \arctan \frac{-z}{-w_{1}-x}-2 \arctan \frac{-h_{\mathrm{pm}}-z}{-w_{1}-x}+2 \arctan \frac{-h_{\mathrm{pm}}-z}{-w_{2}-x}-2 \arctan \frac{-z}{-w_{2}-x}\right]
\end{aligned}
$$

The magnetic flux density distributions for the-surface mounted PMG (\#1) and flux concentrated PMG (\#2) can also be obtained using this method. Fig. 4 plots the calculated and measured transversal distributions (along the $x$ axis) of $B_{\mathrm{x}}$ and $B_{\mathrm{z}}$ at the height $z=0.001 \mathrm{~m}$ from the upper surface of the \#1 and \#2 PMGs, respectively. The PMG filed was measured by sweeping a Hall probe along the transversal direction. It can be seen that the theoretical results are consistent with the experimental measurements, where the small error is mainly caused by ignoring the influence of the soft iron on the flux density distribution. This validates the accuracy of the models built by the ECS method.

Based on the ECS model described in Eqs.(3) and (4), the transversal distributions of flux density components $B_{\mathrm{x}}$ and $B_{\mathrm{z}}$ of the Halbach array PMG are shown in Figs. 5(a) and (b), respectively. As shown, the amplitudes and gradients of the magnetic flux densities decrease as $h$ increases, and the generated $B_{\mathrm{x}}$ and $B_{\mathrm{z}}$ have been substantially improved compared to the cases of \#1 and \#2 PMG.

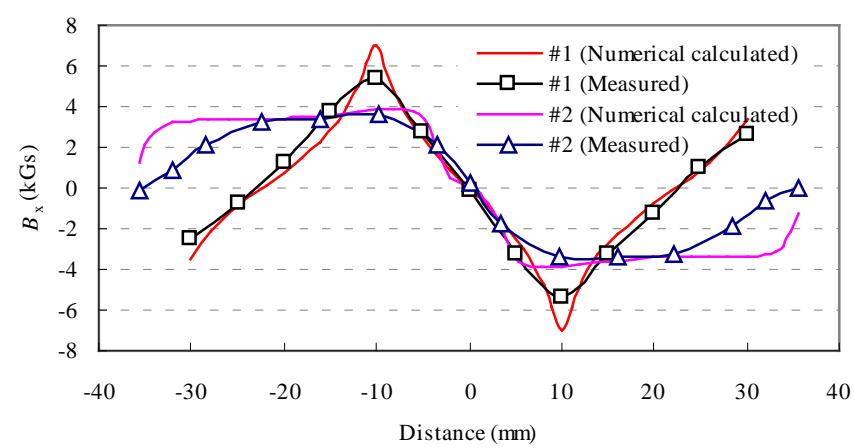

(a)

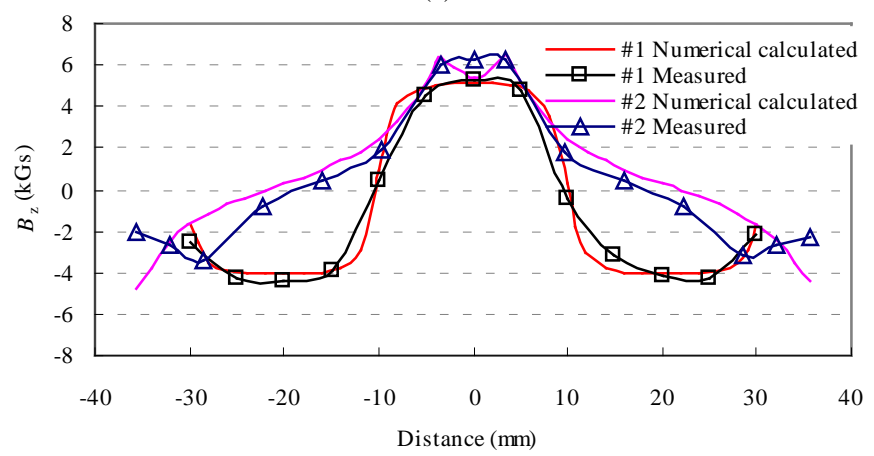

(b)

Fig. 4. Calculated and measured flux density distributions of PMGs (\#1 and \#2). (a) $B_{x}$ along the $x$ axis at $z=1 \mathrm{~mm}$.(b) $B_{z}$ along $x$ axi at $z=1 \mathrm{~mm}$.

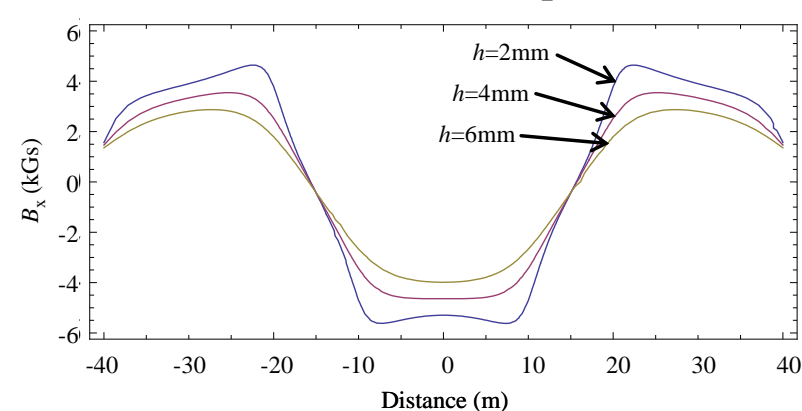

(a)

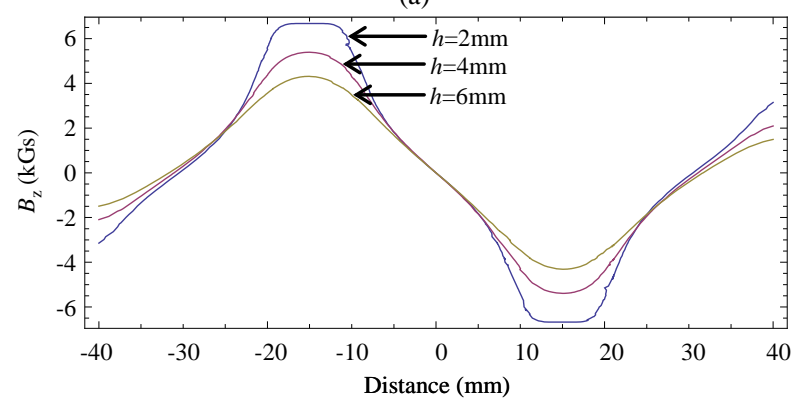

(b)

Fig. 5. Transversal distributions of flux density-at different heights ( $z$ ) of the Halbach array PMG (\#3). (a) $B_{\mathrm{x}}$ along the $x$ axis at different heights. (b) $B_{\mathrm{z}}$. along the $x$ axis at different heights $(z)$.

\subsection{Levitation and guidance forces}

Based on the magnetic flux density distributions of the PMGs, the levitation and guidance forces, between a rectangle HTS bulk and the PMG can be calculated as follows

$$
\begin{aligned}
& F_{\text {Lev }}=\int_{0}^{h_{s}} \int_{l_{s} / 2}^{l_{s} / 2-\delta} \int_{w_{s} / 2}^{w_{s} / 2-\delta} J_{c} \times B_{x} \mathrm{~d} x \mathrm{~d} y \mathrm{~d} z \\
& F_{\text {Gui }}=\int_{0}^{h_{s}} \int_{l_{s} / 2}^{l_{s} / 2-\delta} \int_{w_{s} / 2}^{w_{s} / 2-\delta} J_{c} \times B_{z} \mathrm{~d} x \mathrm{~d} y \mathrm{~d} z
\end{aligned}
$$

where $h_{s}, l_{s}$, and $w_{\mathrm{s}}$ are the height, length and width of the HTS bulk, respectively, $\delta$ the depth of field penetration calculated by $\delta=\left(B_{z}-B_{\text {trap }}\right) / \lambda \mu_{0} J_{c}$ [19], $B_{\text {trap }}$ the trapped magnetic flux density in the HTS bulk, $\lambda$ the Nagaoka coefficient determined by the configuration of a sample, and $J_{\mathrm{C}}$ the critical current density.

For the three types of PMGs, the $F_{\mathrm{Lev}}$ of them are calculated as listed in Table 3, and the ratios of $F_{\mathrm{Lev}} / V_{\mathrm{pm}}$ are also calculated respectively, where $V_{\mathrm{pm}}$ is the volume of PM used in the PMG. From Table 3, the Halbach array PMG (\#3) has the biggest $F_{\text {Lev }}$ and $F_{\text {Lev }} /$ $V_{\mathrm{pm}}$. So the Halbach array PMG can provide the optimum system performance with better ratio (levitation force) / (PM volume) or Newtons per cubic centimeter. However, the flux concentration PMG (\#2) uses less PMs and is readily to assemble to experiment, and selected here for the practical prototype. 
Table 3

Levitaion forces of the three PMGs

\begin{tabular}{cccc}
\hline \hline PMG & $F_{\text {Lev }}(\mathrm{N} / \mathrm{m})$ & $V_{\mathrm{pm}}\left(\mathrm{cm}^{3} / \mathrm{m}\right)$ & $F_{\mathrm{Lev}} / V_{\mathrm{PM}}$ \\
$\# 1$ & 520.7 & 15 & 34.7 \\
$\# 2$ & 615 & 10 & 61.5 \\
$\# 3$ & 1261.3 & 16 & 78.8 \\
\hline \hline
\end{tabular}

\section{Model of HTSLSM}

Fig.6 plots the flux linkage distribution on a longitudinal section of the HTSLSM obtained by the Maxwell/Ansoft ${ }^{\mathrm{TM}}$ 2D finite element analysis. As shown, the majority of magnetic flux flows along the iron core in the primary or stator because of the high permeability, and the flux path is in a reasonably regular pattern. According to the empirical value, the relative permeability of HTS bulk magnet $\mu_{\mathrm{r}}$ is set to be 0.4 . Considering the reluctances of the HTS bulk magnets and air gaps and neglecting the magnetic reluctance of the primary iron core, the magnetic flux linkage of one coil due to one pole-pair HTS bulk magnets can be obtained approximately as

$$
\begin{aligned}
\psi_{\text {SC }} & =\frac{F}{R_{\text {tot }}}=\frac{2 h_{\mathrm{s}}}{\frac{\mu_{\mathrm{r}} \mu_{0} l_{\mathrm{s}} w_{\mathrm{s}}}{2}+\frac{2 g}{\mu_{0} \cdot\left(l_{\mathrm{s}}+l_{\mathrm{t}}\right) / 2 \cdot\left(w_{\mathrm{s}}+w_{\mathrm{t}}\right) / 2}+\frac{2 \tau}{\mu_{0} \cdot w_{\mathrm{s}} \cdot h_{\mathrm{s}}}} \\
& =\frac{B \cdot h_{\mathrm{s}}\left(l_{\mathrm{s}}+l_{\mathrm{t}}\right) \cdot\left(w_{\mathrm{s}}+w_{\mathrm{t}}\right) l_{\mathrm{s}} w_{\mathrm{s}} h_{\mathrm{s}}}{h_{\mathrm{s}}\left(l_{\mathrm{s}}+l_{\mathrm{t}}\right) \cdot\left(w_{\mathrm{s}}+w_{\mathrm{t}}\right) h_{\mathrm{s}}+4 \mu_{\mathrm{r}} g l_{\mathrm{s}} w_{\mathrm{s}} h_{\mathrm{s}}+\mu_{\mathrm{r}} \tau l_{\mathrm{s}}\left(l_{\mathrm{s}}+l_{\mathrm{t}}\right) \cdot\left(w_{\mathrm{s}}+w_{\mathrm{t}}\right)}
\end{aligned}
$$

where $F$ is the magnetomotive force of magnetic circuit, $R_{\text {tot }}$ the total magnetic reluctance, $H_{\mathrm{c}}$ the magnetic field intensity of HTS bulk magnet, $\tau$ the pole pitch, $g$ the air gap length, $l_{\mathrm{s}}, w_{\mathrm{s}}$, and $h_{\mathrm{s}}$ the length, width and height of the HTS bulk magnet, and $l_{\mathrm{t}}$ and $w_{\mathrm{t}}$ the length and width of stator-tooth, respectively. Substituting the parameters listed in Table 1 into Eq.7, it can get the value of $\psi_{\mathrm{sc}}$ as $0.42 \mathrm{mWb}$.

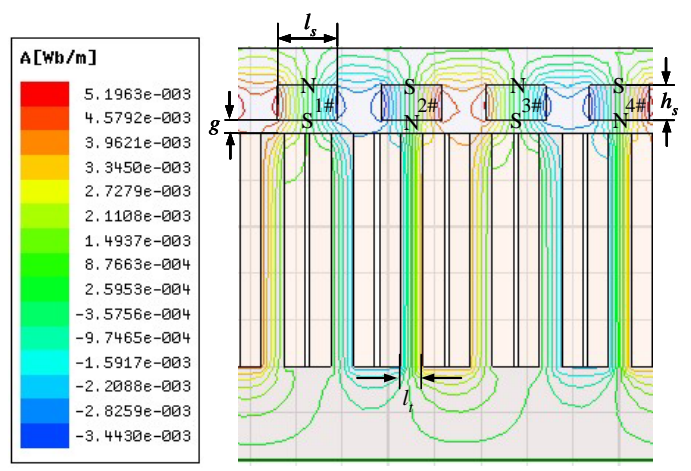

Fig. 6. Flux linkage of the HTSLSM.

The root means square (RMS) value of the back electromotive force (EMF) can be determined by $E_{0}=\pi N_{1} k_{N} \psi_{\mathrm{SC}} \nu_{\mathrm{s}} / \sqrt{2} \tau$, and then the electromagnetic thrust $F_{\text {em }}$ by

$$
\begin{aligned}
F_{\mathrm{em}} & =\frac{P_{\mathrm{em}}}{V_{\mathrm{s}}}=\frac{3 P E_{0}\left[U\left(X_{\mathrm{t}} \sin \theta+R_{1} \cos \theta\right)-E_{0} R_{1}\right]}{2 \tau f\left(R_{1}^{2}+X_{\mathrm{t}}^{2}\right)} \\
& =3 P E_{0}\left[U \sqrt{R_{1}^{2}+X_{\mathrm{t}}^{2}} \sin (\theta+\phi)-E_{0} R_{1}\right] / 2 \tau f\left(R_{1}^{2}+X_{\mathrm{t}}^{2}\right)
\end{aligned}
$$

where $P_{\mathrm{em}}$ is the electromagnetic power, $v_{\mathrm{s}}$ the synchronous velocity equal to $2 \tau f$, $f$ the frequency, $N_{1}$ the number of turns of winding, $k_{\mathrm{N}}$ the winding factor, $R_{1}$ the phase resistance, $X_{\mathrm{t}}$ the synchronous reactance, $P$ the number of pole pairs, $U$ the phase voltage, $\phi$ the load shift angle, and $\phi=\arctan R_{1} / X_{\mathrm{t}}$. When the load angle $\theta=90^{\circ}-\phi$, the maximum electromagnetic thrust can be obtained as

$$
F_{\text {em_max }}=\frac{3 p E_{0}\left(U \sqrt{R_{1}^{2}+X_{\mathrm{t}}^{2}}-E_{0} R_{1}\right)}{2 \tau f\left(R_{1}^{2}+X_{\mathrm{t}}^{2}\right)}
$$

Fig.7 illustrates the $F_{\text {em max }}$ versus the trapped flux density $B_{\text {trap }}$ of the HTS bulk magnets by different excitations of $U / f$ when $f=5 \mathrm{~Hz}$. It is shown that $F_{\text {em_max }}$ can reach $3.8 \mathrm{kN}$ if $B_{\text {trap }}=3 \mathrm{~T}$ and $U / f=40$.

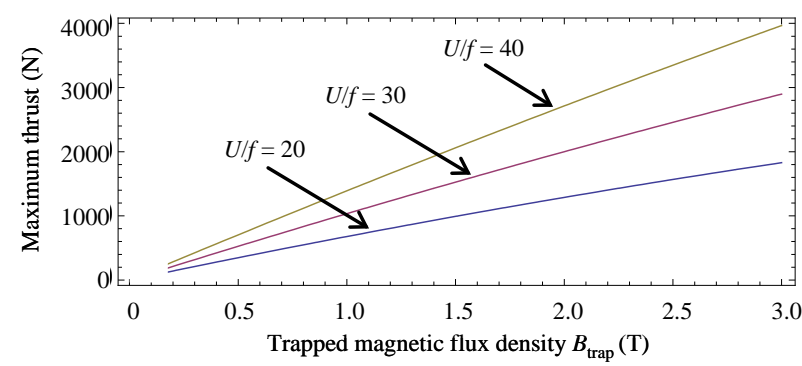

Fig. 7. Maximum thrust versus trapped magnetic flux density for different $U / f$.

\section{Magnetization Characteristics of HTS Bulk Magnet}

Fig.8 shows the measured magnetic flux trapping characteristics of HTS bulk magnets obtained by the field-cooled (FC) magnetization method with a DC magnet, and the ZFC magnetization methods with a DC magnet and a pulse magnetizer, respectively. As shown in the figure, in all three cases, the $B_{\text {trap }}$ of the HTS bulk magnet increases linearly with the external magnetic flux density, $B_{\text {ext }}$, before saturation The amplitude of $B_{\text {ext }}$ obtained by using the ZFC pulse magnetization is about twice as that by the ZFC DC magnetization, and four times as that by the FC DC magnetization. The results are very useful to select the appropriate methods to obtain HTS bulk magnets for different practical applications. For the low-field magnetization, although the FC DC magnetization is feasible, it is favorable to choose the ZFC pulse magnetization for its higher magnetization field and lower cost.

The trapping magnetic flux characteristics by ZFC pulse magnetization for three HTS bulk samples (BU 1, BU 2 and BU 3) having different qualities are given in Fig. 9. It can be seen that the quality of HTS bulk has substantial influence on the magnetic flux trapping capability. The optimum value of pulse $B_{\text {ext }}$ for saturation magnetization with maximum trapped flux $B_{\text {trap } \max }$ increases with the quality of HTS bulk, and finally reaches a steady value of $1.1 \mathrm{~T}$ for the samples used. An optimum $B_{\text {ext }}$ can hence be found to magnetize the HTS bulks.

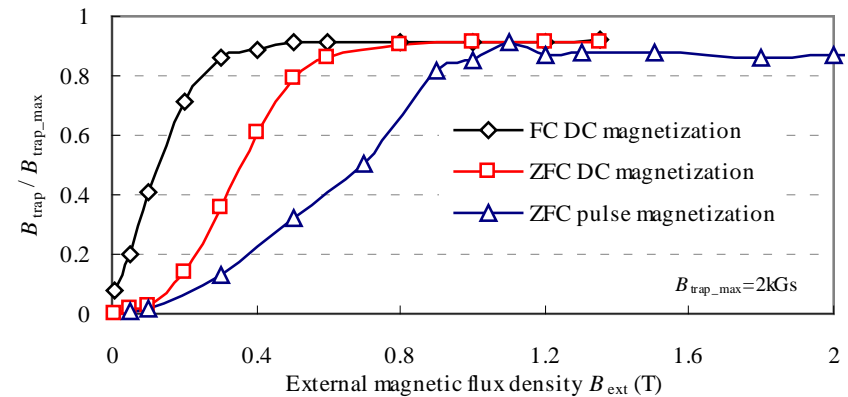

Fig. 8. Comparison of HTS bulk magnetic flux trapping characteristics within different magnetization methods.

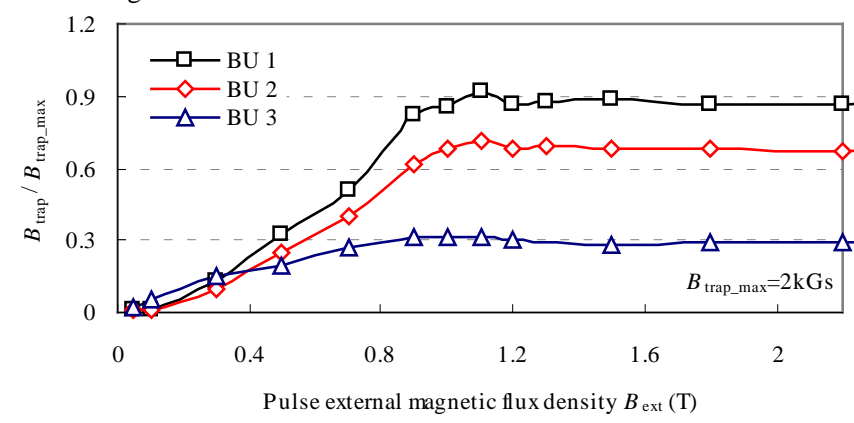

Fig. 9. Trapped magnetic flux density versus pulse $B_{\text {ext }}$ for different samples.

After trapping magnetic flux, it is also important to understand the flux distribution characteristics of the HTS bulk magnets for practical applications. An analytical model of the magnetic field distribution at an arbitrary point $(r, z)$ for a disk-shaped HTS bulk magnet with a constant $J_{\mathrm{C}}$ is built by [20] 


$$
B_{z}(r, z)=\frac{\mu_{0} J_{c}}{2 \pi} \int_{0}^{R} \int_{z}^{D+z} \frac{K(k)+\frac{a_{s}^{2}-r^{2}-z_{s}^{2}}{\left(a_{s}+r\right)^{2}+z_{s}^{2}} E(k)}{\sqrt{\left(a_{s}+r\right)^{2}+z_{s}^{2}}} \mathrm{~d} z_{s} \mathrm{~d} a_{s}
$$

where $k^{2}=4 a_{s} r\left[\left(a_{s}+r\right)^{2}+z_{s}^{2}\right]^{-1}, K$ and $E$ are of the first and second kind complete elliptic integrals, $R$ and $D$ the radius and thickness of the disk-shaped HTS bulk magnet, respectively. In the case of $r=0$, the magnetic field $B_{z}(z)$ can be simplified as

$$
B_{z}(z)=\frac{\mu_{0} J_{c}}{2}\left((z+D) \ln \frac{R+\sqrt{R^{2}+(z+D)^{2}}}{z+D}-z \ln \frac{R+\sqrt{R^{2}+z^{2}}}{z}\right)
$$

For an HTS bulk sample with a diameter of $26 \mathrm{~mm}$, Fig.10 shows the magnetic flux density versus the distance from the upper surface of HTS bulk magnet along the axis line. As shown, a higher $J_{\mathrm{c}}$ would result in a higher $B_{\text {trap. }}$. When $J_{\mathrm{c}}=2 \times 10^{8} \mathrm{~A} / \mathrm{m}^{2}$, the maximum central $B_{\text {trap }}$ can reach $1.1 \mathrm{~T}$ at $77 \mathrm{~K}$.

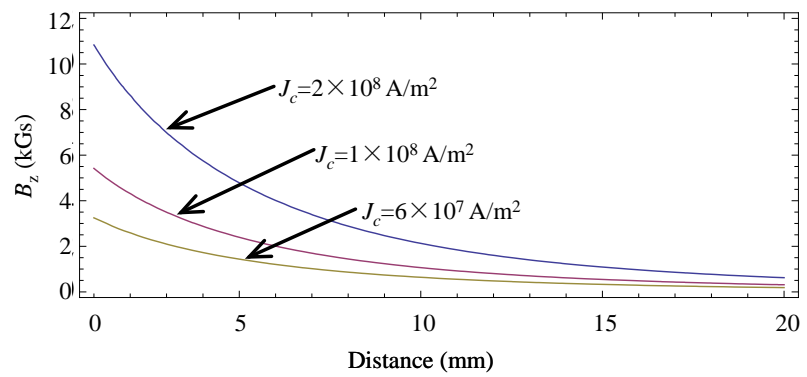

Fig. 10. $B_{z}(z)$ versus distance (z) for different $J_{\mathrm{c}}$ where the diameter of HTS bulk $\phi=26 \mathrm{~mm}$.

\section{Experimental Verification}

Fig. 11 shows the prototype of an HTSLSM with HTS bulk magnet secondary (mover) for the HTS magnetic suspension and propulsion system. The machine is controlled by the technique of space vector pulse width modulation (SVPWM). The locked-rotor thrust of the HTSLSM can be measured by using a pulling force sensor connected to one side of the mover, and the load connected to the other side of the mover as shown in Fig. 12. The range of the pulling force sensor is $0-2000 \mathrm{~N}$ matched the output voltage of 1 - 5 V. In practical thrust measurements, PMs are used to form an equivalent magnetic circuit for the tests.

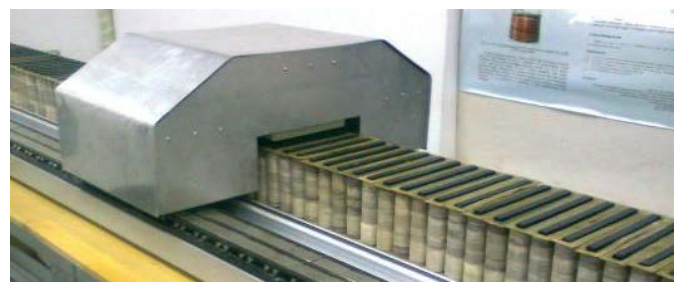

Fig. 11. Prototype of the HTSLSM for an HTS magnetic suspension and propulsion system.

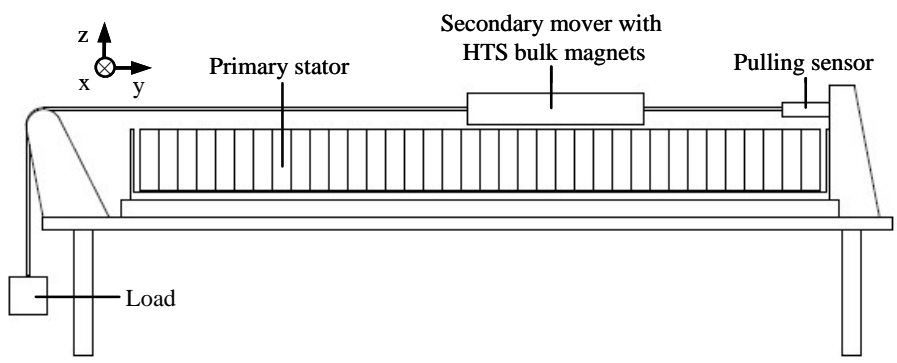

Fig. 12. Thrust measurement system for the HTSLSM.

Fig.12-3 illustrates the locked-mover thrusts measured in two cases: (a) $f=2 \mathrm{~Hz}, U / f=15$, air gap length $g=12.0 \mathrm{~mm}$, and (b) $f=10$ $\mathrm{Hz}, U / f=12, g=13.0 \mathrm{~mm}$. The waveforms of the locked-mover thrusts are nearly sinusoidal, and the their amplitudes are the maximum thrusts $F_{\text {em_max }}$. Hence, the maximums in two excitations are
98.9 $\mathrm{N}$ and $418.9 \mathrm{~N}$, respectively. Because the force of gravity of the applied load is $156.0 \mathrm{~N}$, the maximum opposite pulling force of the sensor to measure is thus limited to $156.0 \mathrm{~N}$. This is why the inverse maximum thrusts shown in Fig.12-3 (b) are smaller than the positive amplitudes.

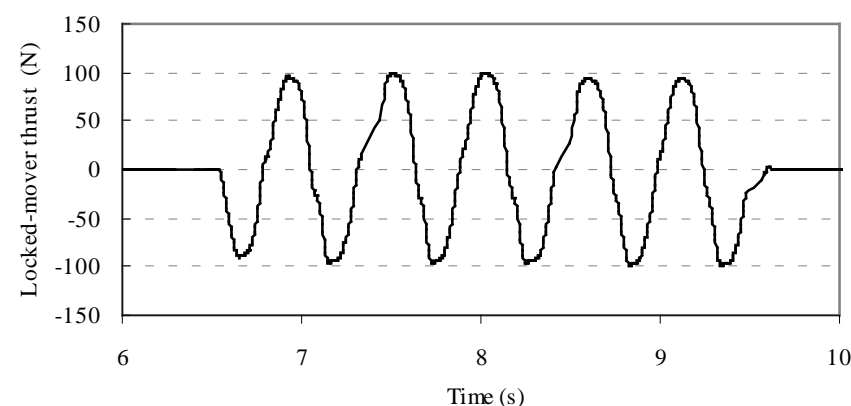

(a)

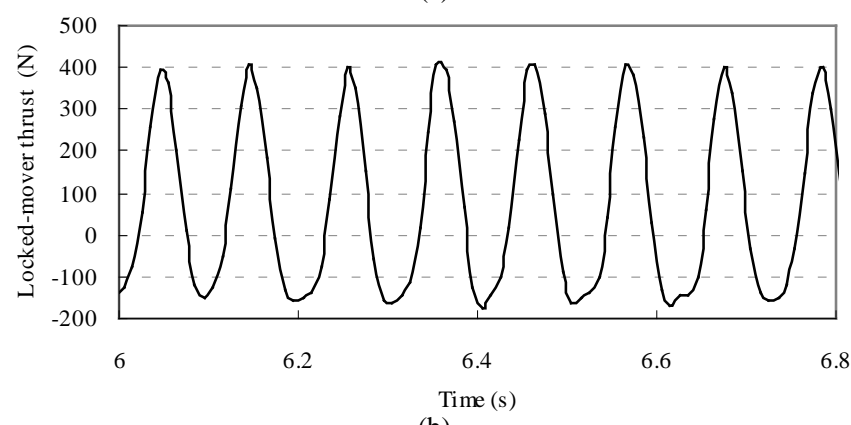

(b)

Fig. 123. Locked-mover thrust, (a) $f=2 \mathrm{~Hz}, U / f=15, g=12 \mathrm{~mm}$, (b) $f=10 \mathrm{~Hz}$, $U / f=12, g=13 \mathrm{~mm}$.

Based on the control platform developed by LabVIEW ${ }^{\mathrm{TM}}$, the stator phase currents have been measured with different $f$ and $U / f$ as shown in Fig. 13-4. In these testing, the PWM period $T_{\mathrm{s}}$ is set to $0.0025 \mathrm{~s}$, the dead time $10 \mu \mathrm{s}$, and the DC Bus voltage $200 \mathrm{~V}$. As seen from this figure, the stator phase current $I$ increases with $f$ initially, and then decreases after reaching the maximum $I_{\max }$, where the frequency is defined the optimum frequency $f_{\mathrm{o}}$. It can be seen that $I_{\max }$ increases as $U / f$ grows up, while $f_{0}$ could decrease.

Fig.14-15 shows the measured $F_{\text {em_max }}$ versus $f$ under different $U / f$ when the $g=12.0 \mathrm{~mm}$. From the figure, $F_{\mathrm{em} \_ \text {max }}$ increase with $f$ initially and then decrease after reaching their —peak values of 285.8 $\mathrm{N}$ and $403.5 \mathrm{~N}$ at $U / f=5$, -at $f=13 \mathrm{~Hz}$, and $U / f=10$, -at $f=11 \mathrm{~Hz}$, respectively;.

Fig.15-6 depicts the measured $F_{\text {em_max }}$ versus air gap length $g$ under different $f$ and $U / f$. As shown, $F_{\mathrm{em} \_ \text {max }}$ decreases linearly as the $g$ increases. In order to operate the system at the rated thrust, it is necessary to keep the length of air gap of the HTS magnetic suspension at a constant value.

Fig.16-7 plots the measured $F_{\text {em_max }}$ versus $I$. As shown, $F_{\text {em_max }}$ increases almost linearly with $I$. Experiment agrees well with simulation based on the magnetic circuit method except for a few points. These errors are mainly caused by the magnetic saturation in the primary stator iron core.

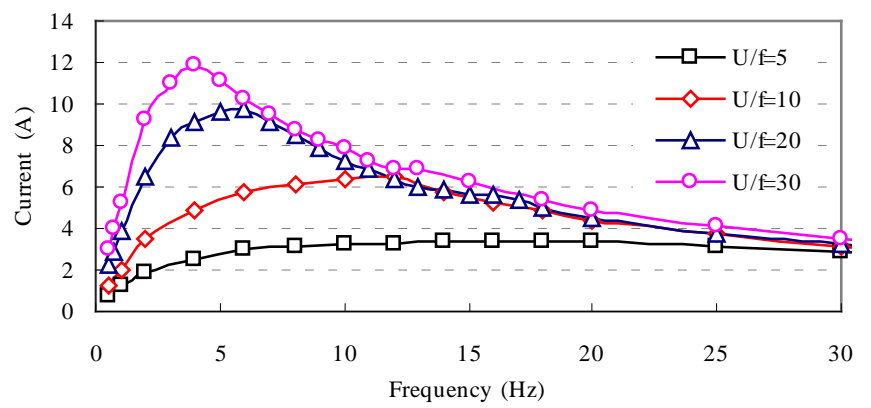

Fig. 134. Stator phase current versus frequency with different $U / f$. 


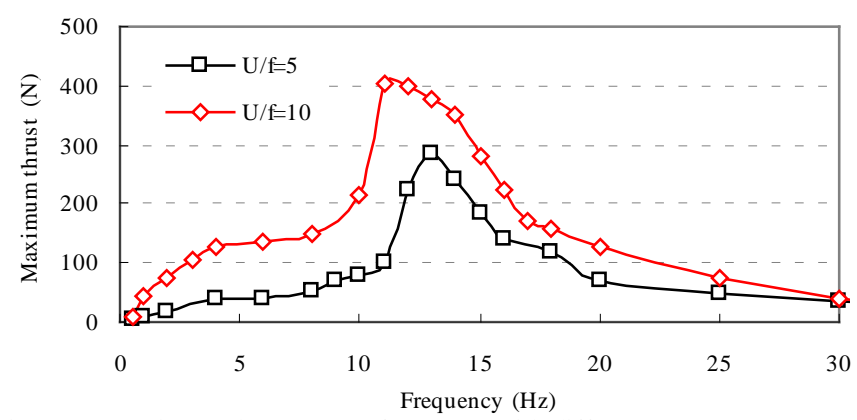

Fig. 145. Maximum thrust versus frequency with different $U / f(g=12 \mathrm{~mm})$.

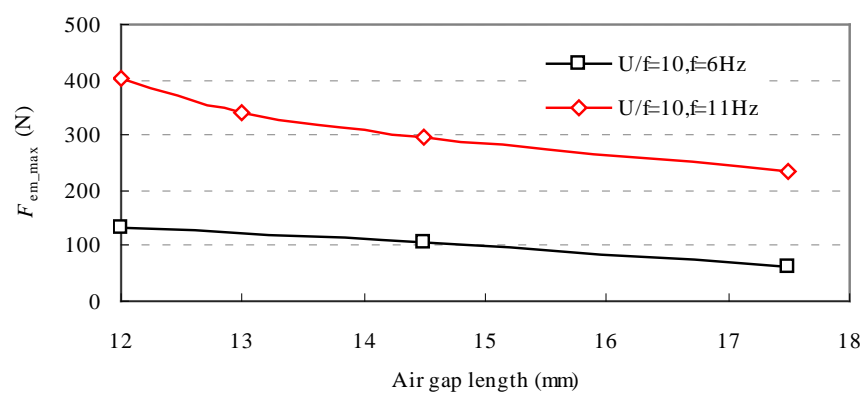

Fig. 15-6. Maximum thrust versus air gap length with different $f$ and $U / f$.

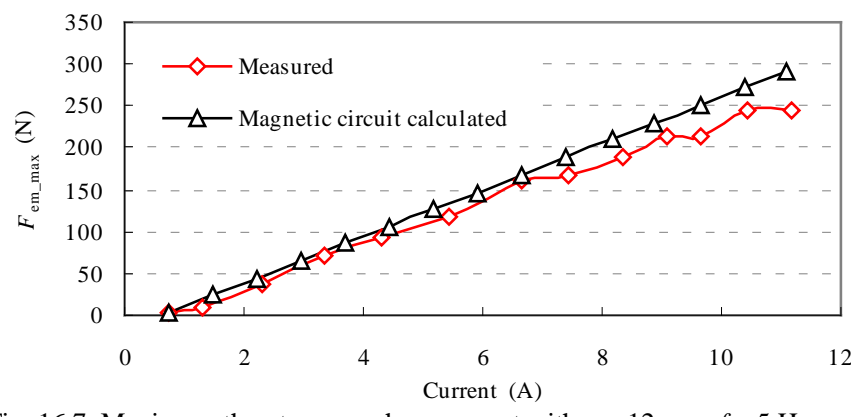

Fig. 16 7. Maximum thrust versus phase current with $g=12 \mathrm{~mm}, f=5 \mathrm{~Hz}$.

\section{Conclusions}

An HTS magnetic suspension and HTSLSM propulsion system has been developed in this paper. Three types of PMGs are-were firstly analyzed by using the equivalent current sheet method respectively, and then the levitation force are calculated based on them having the results show that the Halbach array PMG can provide the largest levitation force and best ratio (levitation force) / (permanent magnet volume). That is to say, the Halbach array PMG can provide the optimum system performance. The magnetization characteristics of HTS bulk based on the different methods have been exprimental studied, and the conclusions form a reference to select an optimal magnetization method to obtain HTS bulk magnets. The numerical model of the HTSLSM has been built up and the experiments validation for its thrust performance have been comprehensively carried out with the results show that the maximum thrust of the HTSLSM increases linearly with phase current, decreases linearly with the air gap length, and increases with the frequency initially and then decreases with it after reaching its peak value, which reflect the characteristics of HTSLSM and the developed control system, and it benefits the optimal electromagnetic design and the control scheme development of an HTSLSM for the HTS magnetic suspension application.

\section{References}

[1] S. Kusada, M. Igarashi, K. Nemoto, T. Okutomi, S. Hirano, K. Kuwano, T. Tominaga, M. Terai, T. Kuriyama, K. Tasaki, T. Tosaka, K. Marukawa, S. Hanai, T. Yamashita, Y. Yanase, H. Nakao, M. Yamaji, The project overview of the HTS magnet for superconducting maglev, IEEE Trans. Appl. Superconduct. 17 (2007) 2111-2116.

[2] K. Kuwano, M. Igarashi, S. Kusada, K. Nemoto, T. Okutomi, S. Hirano, T. Tominaga, M. Terai, T. Kuriyama,; K. Tasaki, T. Tosaka, K. Marukawa, S. Hanai, T. Yamashita, Y. Yanase, H. Nakao, M. Yamaji, The running tests of the superconducting maglev using the HTS magnet, IEEE Trans. Appl.
Superconduct. 17 (2007) 2125-2128.

[3] K. Tasaki, K. Marukawa, S. Hanai, T. Tosaka, T. Kuriyama, T. Yamashita, Y. Yanase, M. Yamaji, H. Nakao, M. Igarashi, S. Kusada, K. Nemoto, S. Hirano, K. Kuwano, T. Okutomi, M. Terai, HTS magnet for maglev applications (1)—coil characteristics, IEEE Trans. Appl. Superconduct. 16 (2006) 1100-1103.

[4] M. Terai, M. Igarashi, S. Kusada, K. Nemoto, T. Kuriyama, S. Hanai, T. Yamashita, H. Nakao, The R\&D project of HTS magnets for the superconducting maglev, IEEE Trans. Appl. Superconduct. 16 (2006) 1124-1129.

[5] C.Y. Lee, J.M. J. M. Jo, Y.J. Han, D.K. Bae, Y.S. Yoon, S. Choi, D.K. Park, T.K. Ko, Estimation of current decay performance of HTS electromagnet for maglev, IEEE Trans. Appl. Superconduct. 20 (2010) 907-910.

[6] C. Gu, M.L. Liu, H.W. Xing, T. Zhou, W.S. Yin, J. Zong, Z.H. Han, Design, construction and performance of an EMS-based HTS maglev vehicle, Physica C 423 (2005) 37-44.

[7] J.S. Wang, S.Y. Wang, Y. Zeng, H.Y. Huang, F. Luo, Z.P. Xu, Q.X. Tang, G.B. Lin, C.F. Zhang, Z.Y. Ren, G.M. Zhad, D.G. Zhu, S.H. Wang, H. Jiang, M. Zhu, C.Y. Deng, P.F. Hu, C.Y. Li, F. Lib, J.S. Lian, X.R. Wang, L.H. Wang, X.M. Shen, X.G. Dong, The first man-loading high temperature superconducting maglev test vehicle in the world, Physica $\mathrm{C}$ 378-381 (2002) 809-814.

[8] L. Schultz, O.de Haas, P. Verges, C. Beyer, S. Rohlig, H. Olsen, L. Kühn, D. Berger, U. Noteboom, U. Funk, Superconductively levitated transport system - the SupraTrans project, IEEE Trans. Appl. Superconduct. 15 (2005) 2301-2305.

[9] K.L. Kovalev, S.M.-A. Koneev, V.N. Poltavec, W. Gawalek, Magnetically levitated high-speed carriages on the basis of bulk HTS elements, in Pro. 8th Intern. Symp. Magn. Susp. Technol. (ISMST’8), Dresden, Germany, 2005, p. 51.

[10] K. Yoshida, H. Matsumoto, M. Eguchi, Optimal design of thrust force in vertical-type HTS bulk LRM, Physica C 426-431 (2005) 839-847.

[11] R. Muramatsu, S. Sadakata, M. Tsuda, A. Ishiyama, Trial production and experiments of linear actuator with HTS bulk secondary, IEEE Trans. Appl. Superconduct. 11 (2001) 1976-1979.

[12] G. Stumberger, M.T. Aydemir, A.L. Thomas, Design of a linear bulk superconductor magnet synchronous motor for electromagnetic aircraft launch systems, IEEE Trans. Appl. Superconduct. (2004) 54-62.

[13] L.H. Zheng, J.X. Jin, Y.G. Guo, H.Y. Lu, J.G. Zhu, Design and electromagnetic analysis of a HTS linear synchronous motor, 2009 International Conference on Applied Stperconductivity and Electromagnetic Devices (ASEMD), Chengdu, China, 2009, p. 5.

[13] J. X. Jin, L. H. Zheng, W. Xu, Y. G. Guo, J. G. Zhu, Thrust characteristic of a double-sided HTSLSM with an HTS magnetic suspension system, J. Appl. Phy. 109(2011) 073916-1-073916-4.

[14] J. X. Jin, L. H. Zheng, Driving models of high temperature superconducting linear synchronous motors and characteristic analysis, Superconduct. $\quad$ Sci. $\quad$ Tech. 24 (2011) DOI: 10.1088/0953-2048/24/5/055011.

[145] A. Takahashi, H. Ueda, A. Ishiyama, Trial production and experiment of linear synchronous actuator with field-cooled HTS bulk secondary, IEEE Trans. Appl. Superconduct. 13 (2003) 2251-2254.

[15-6] A. Sato, H. Ueda, A. Ishiyama, Operational characteristics of linear synchronous actuator with field-cooled HTS bulk secondary, IEEE Trans. Appl. Superconduct. 15 (2005) 2234-2237.

[16-7] W.S. Kim, S.Y. Jung, H.Y. Choi, H.K. Jung, J.H. Kim, S.Y. Hahn, Development of a superconducting linear synchronous motor, IEEE Trans. Appl. Superconduct. 22 (2002) 842-845.

[18] T. Kikuma and A.Ishiyama, Improvement of superconducting cylindrical linear induction motor, IEEE Trans. Appl. Superconduct. 11 (2011) 2331-2334

[17-9] T. Suzuki, S. Araki, K. Koibuchi, K. Ogawa, K. Sawa, K. Takeuchi, M. Murakami, K. Nagashima, H. Seino, Y. Miyazaki, N. Sakai, I. Hirabayashi, Y. Iwas, A study on levitation force and its time relaxation behavior for a bulk superconductor-magnet system, Physica C 468 (2008) 1461-1464.

[1820] M. Sawamura, M. Morita, H. Hirano, Magnetic properties of Ag-added Gd-Ba-Cu-O superconductors, Physica C 392-396 (2003) 531-534. 
Fig. 1. Schematic illustration of the HTS magnetic suspension and propulsion system with an HTSLSM drive.

Fig. 2. Schematic structures of (a) Surface mounted PMG (\#1), (b) Flux concentration PMG (\#2), and (c) Halbach array PMG (\#3).

Fig. 3. Current sheet models, (a) Surface mounted PMG (\#1), (b) Flux concentration PMG (\#2), and (c) Halbach array PMG (\#3).

Fig. 4. Calculated and measured distributions of (a) $B_{x}$ and (b) $B_{z}$ along the X axis at $h=1 \mathrm{~mm}$ or $z=1 \mathrm{~mm}$ of the surface mounted and flux concentration PMGs.

Fig. 5. Transversal distributions of flux density (a) $B_{*}$ and (b) $B_{z}$ along the $X$ axis at different heights ( $\mathrm{z}$ ). at different heights (z) of the Halbach array PMG (\#3): (a) $B_{x}$ and (b) $B_{\mathrm{z}}$

Fig. 6. Flux linkage of the HTSLSM.

Fig. 7. Maximum thrust versus trapped magnetic flux density for different $U / f$.

Fig. 8. HTS bulk magnetic flux trapping characteristics comparison within different magnetization methods.

Fig. 9. Trapped magnetic flux density versus pulse $B_{\text {ext }}$ for different samples.

Fig. 10. $B_{z}(z)$ versus distance $(z)$ for different critical current density $J_{c}$ where the diameter of HTS bulk $\phi=26 \mathrm{~mm}$.

Fig. 11. Prototype of the HTSLSM for an HTS magnetic suspension and propulsion system.

Fig. 12. Thrust measurement system for the HTSLSM.

Fig. 13. Locked-mover thrust, (a) $f=2 \mathrm{~Hz}, \mathrm{U} / \mathrm{f}=15, \mathrm{~g}=12 \mathrm{~mm}$, (b) $\mathrm{f}=10 \mathrm{~Hz}, \mathrm{U} / \mathrm{f}=12, \mathrm{~g}=13 \mathrm{~mm}$.

Fig. 13 4. Stator phase current versus frequency with different $\mathrm{U} / \mathrm{f}$.

Fig. 14 5. Maximum thrust versus frequency with different $\mathrm{U} / \mathrm{f}(\mathrm{g}=12 \mathrm{~mm})$.

Fig. 15-6. Maximum thrust versus frequency differenth air gaplengths (U/f-10)-air gap length under different $U / f$ and $f$.

Fig. 16-7. Maximum thrust versus phase current at different frequencies ( under $g=12 \mathrm{~mm}, f=5 \mathrm{~Hz}$.

Table 1 Main dimensions of transversal section of the PMGs

Table 2. Main parameters of the HTSLSM

Table 3 Levitaion forces of the three PMGs 